\section{Ten key issues in modern flow chemistry}

\author{
Jens Wegner, Sascha Ceylan and Andreas Kirschning* \\ DOI: $10.1039 / \mathrm{c0cc05060a}$
}

Ten essentials of synthesis in the flow mode, a new enabling technology in organic chemistry, are highlighted as flashlighted providing an insight into current and future issues and developments in this field.

\section{Why flow? An effort to provide a brief introduction}

For chemists from Liebig's time being transferred into a common synthetic laboratory of the 20th century, most equipment would look very familiar

Institute of Organic Chemistry and Center of Biomolecular Drug Research (BMWZ),

Leibniz Universität Hannover,

Schneiderberg 1b, 30167 Hannover, Germany. E-mail: andreas.kirschning@oci.uni-hannover.de; Fax: + 49 (0)511 7623011;

Tel: $+49(0) 5117624614$ today. We all work with standardised glassware, use somewhat sophisticated ways of stirring and after a battle of at least two decades we accept that microwave radiation can beneficially be added to the portfolio of classical heating devices. ${ }^{1}$ Indeed chemists have mainly been focused on the chemistry occurring inside the flasks or reaction vessels and they have done so in a very elaborated and successful manner. Despite their many advantages continuous flow processes have commonly flourished only in the industrial environment of chemical and biotechnological production. ${ }^{2}$ Several advantages have been recognised such as facile automation, secured reproducibility, improved safety and process reliability. Indeed, with continuous flow processes constant reaction parameters (temperature, time, amount of reagents and solvents, efficient mixing, etc.) can easily be assured. Over the past 15 years, miniaturisation has entered the field of flow chemistry and modern developments commonly deal with micro- and microfluidic (or mesofluidic) flow devices (see issue 1 below). ${ }^{3}$ With

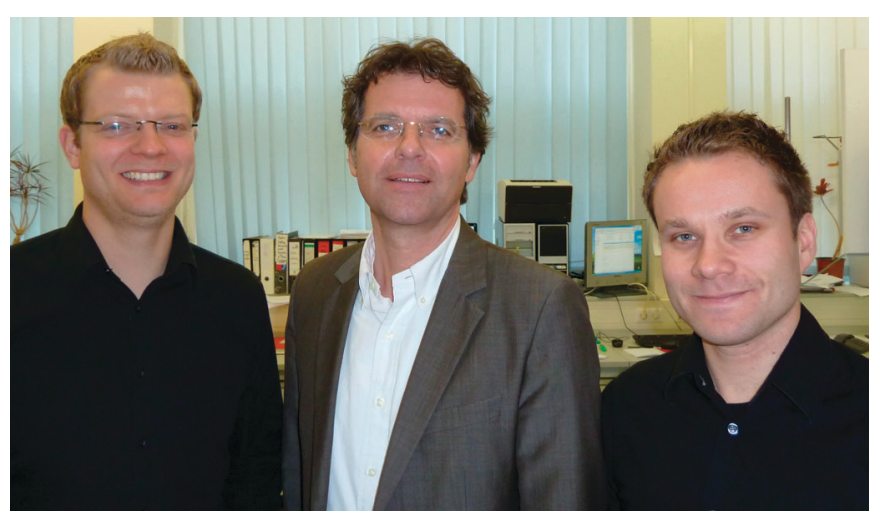

Jens Wegner, Andreas Kirschning and Sascha Ceylan

Jens Wegner (left) was born in 1982 in Nordhorn, Germany. He studied chemistry at the Leibniz University Hannover where he received his diploma degree in 2008. He is currently performing his doctoral studies at Hannover under the supervision of Prof. Andreas Kirschning with the aid of a scholarship by the Fonds der Chemischen Industrie. In 2010 he spent several months in the group of Prof. S. V. Ley at Cambridge University $(U K)$. His research is focussed on the development of an inductively heated micro flow system for organic synthesis. A key aspect of his work is the implementation of a multistep reaction and an immobilised catalyst into micro flow systems.

Sascha Ceylan (right) was born in Augsburg, Germany, in 1981 and studied chemistry at Leibniz University Hannover and at Imperial College London under the guidance of Prof. A. G. M. Barrett (UK). He received his diploma degree in 2007 and thereafter began his doctoral studies in Hannover under the supervision of Prof. A. Kirschning. Following a research stay at the University of Hong Kong (Prof. P. Toy) he received his PhD early 2011. His scientific interest focuses on developing micro flow systems, especially inductively heated reactors, and investigations on palladium catalysed Umpolung reactions.

Andreas Kirschning studied chemistry at the University of Hamburg and Southampton University (UK). In Hamburg, he joined the group of Prof. E. Schaumann and received his PhD in 1989 working in the field of organosilicon chemistry. After a postdoctoral stay at the University of Washington (Seattle, USA) with Prof. H. G. Floss, supported by a Feodor-Lynen scholarship of the Alexander-von Humboldt foundation, he started his independent research at the Clausthal University of Technology in 1991, where he finished his habilitation in 1996. In 2000 he moved to the Leibniz University Hannover and became a director of the institute of organic chemistry. He is one of the editors of RÖMPP online, Natural Products Reports and The Beilstein Journal of Organic Chemistry. His research interests cover structure elucidation as well as the total synthesis and mutasynthesis of natural products, biomedical biopolymers, and synthetic technology (solid-phase assisted synthesis, microreactors, inductive heating). 
respect to heat and mass transfer, efficient mixing as well as precise parameter control miniaturised flow devices have proven very beneficial for performing chemistry that is difficult to conduct under batch conditions e.g. with highly reactive reagents or synthesis via unstable intermediates (see issue 4 below). Furthermore, continuous flow reactors are generally smaller than batch reactors but in an ideal setup and under optimised conditions these bench sized reactors are able to produce more product in a given time than an analogous batch reactor (see issue 2 below). ${ }^{4}$ Particularly, chemical engineers and process chemists have stressed that optimised reaction parameters developed in a bench-sized micro- or minifluidic (or mesofluidic) flow device can be transferred directly to large scale production without the need for substantial further optimisation which is in stark contrast to upscaling batch processes (see issue 10 below).
Undoubtedly, flow chemistry will not completely or generally change the way chemists are performing synthesis in the future, despite the fact that the field experiences a dramatic increase in interest both in academia and industry. But flow chemistry will become a common enabling technology for performing synthesis in the laboratory. ${ }^{5}$ It is beyond the scope of this account to provide an exhaustive overview over the rapidly expanding field of flow chemistry. By specifically covering TEN ISSUES of importance, we rather wish to address the latest trends and what we believe are the most important topics to be considered now and in the near future in flow chemistry and bring that to the attention of the broad readership of Chemical Communications. We do so in a purely highlight format and at the same time are fully aware that our perspective is based on our own experience in this field that we gained over the past ten years, thus likely leaving out some other important topics of concern.

\begin{tabular}{|c|c|c|}
\hline \multirow{2}{*}{$\begin{array}{l}\text { Microfluidic } \\
\text { reactor }(10-500 \mu \mathrm{m} \\
\text { i.d.) }\end{array}$} & Advantages & Disadvantages \\
\hline & $\begin{array}{l}\text { high heat transfer surface to } \\
\text { product volume ratios } \\
\text { good heat transfer } \\
\text { capabilities } \\
\text { ideally suited for optimising } \\
\text { reactions conditions } \\
\text { efficient mixing }\end{array}$ & $\begin{array}{l}\text { o micro channels suffer from } \\
\text { restricted flow capacity } \\
\text { high pressure drop } \\
\text { tendency to block }\end{array}$ \\
\hline $\begin{array}{l}\text { Minifluidic reactor } \\
\text { (500 } \mu \mathrm{m} \text { - several } \\
\text { mm i.d.) }\end{array}$ & $\begin{array}{l}\text { improved flow capacities } \\
\text { lower pressure drop } \\
\text { no blocking of channels } \\
\text { preparation of multigram to } \\
\text { multikilogram quantities } \\
\text { posibility to work with } \\
\text { packed bed reactors }\end{array}$ & $\begin{array}{l}\text { lower heat transfer surface } \\
\text { poorer heat transfer } \\
\text { capabilities }\end{array}$ \\
\hline
\end{tabular}

Fig. 1 Micro versus mini (meso) flow reactors.

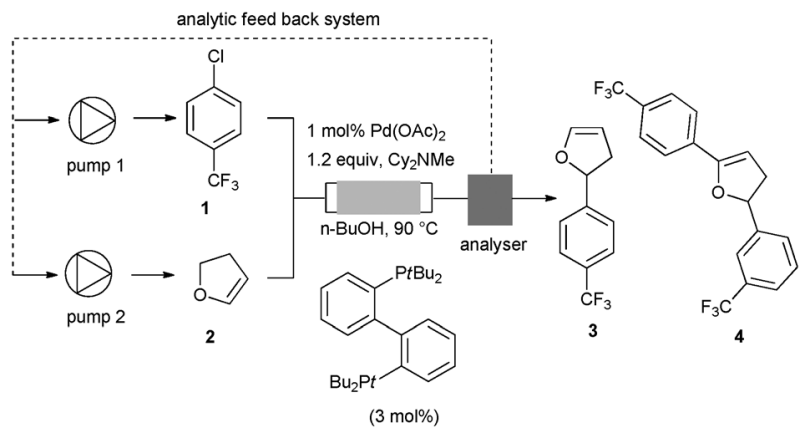

Scheme 1 Self-optimising microfluidic flow system using an inline HPLC analysis.

\section{Issue 1: size matters-micro versus minifluidic (or mesofluidic) reactors}

Common miniaturised bench-sized flow devices rely on reactors that have channels or tubes sized between $10 \mu \mathrm{m}$ to a few $\mathrm{mm}$ in inner diameter. The dimension categorises whether a micro or minifluidic (or mesofluidic) flow reactor is at hand (Fig. 1). ${ }^{6}$

This scale range is sufficiently broad to allow synthesis of approximately $10 \mathrm{mg}$ to an annual production rate of several tens of tons of material of a desired compound. Today microfluidic devices are mainly found in academic laboratories and in analytical and diagnostic applications. Mesofluidic devices are also of academic interest, particularly when combined with functionalised fixed bed materials but have also become popular in the industrial context. ${ }^{7}$ The heat transfer capacity, which is a very important issue for chemical engineers, is heavily influenced by channel size since it determines the heat transfer area per unit volume. For micro reactors parameters are commonly between $5000-50000 \mathrm{~m}^{2} \mathrm{~m}^{-3}$ while for mini reactors the common values are between $100-10000 \mathrm{~m}^{2} \mathrm{~m}^{-3}$. Thus, high heat transfer capacities are achieved with small diameter channels favouring micro over mini flow reactors. But high pressure drops, a limited flow capacity and a tendency to block, are common problems with micro reactors. They are also often fabricated in a manner which makes cleaning and dismantling difficult or impossible. Practically, micro reactors also suffer from the fact that it is very difficult to utilise them for the production of substantial amounts of material. Technically it is difficult to overcome this problem even when massive parallelisation is achieved.

However, the scale of micro flow reactors makes them ideal tools for process development experiments. An illustrative and telling example for advantageously utilising microreactors in practice was recently disclosed by Jensen et al. ${ }^{8}$ The authors set out to rapidly find the ideal parameters for a Heck reaction by designing a self-optimising flow system (Scheme 1). It was known that product 3 readily reacts with an excess of aryl chloride 1 to yield diarylated dihydro furan 4. The analytical "feedback" 
system based on HPLC allowed them to monitor and to individually change the flow rates of the two components being pumped into the microreactor and their initial concentrations in the reaction mixture. Within a short time, optimised conditions at $90{ }^{\circ} \mathrm{C}$ were found $(3: 4=5: 1$, reaction time $5.5 \mathrm{~min})$ which led to a yield of 3 of $82 \%$. Importantly, the authors showed that these conditions could directly be used in a 50-fold larger flow reactor affording similar yields of $\mathbf{3}$.

\section{Issue 2: residence time, flow rate and reactor volume-key factors in flow chemistry}

There are fundamental differences between batch and flow systems with respect to stoichiometry and reaction time. Stoichiometry in batch processes is defined by the concentration of chemical reagents and their volumetric ratio while in flow reactors this is defined by the concentration of reagents and the ratio of their flow rates. In batch production reaction time is determined by how long a vessel is held at a given temperature to reach full conversion. When chemistry is operated under flow conditions the reaction time is determined by the volume of the reactor and the bulk flow rate (Fig. 2, top). Here, it is desirable to reach full conversion inside the reactor with the highest possible flow rate. As the flow rate correlates with the residence time these factors determine how much product is formed in a given time. Under optimised conditions a flow reactor can be operated continuously as long as starting materials are available and are pumped through the reactor. E.g. Hessel and Löwe provided an illustrative example of an industrial batch process, the oxidation of ethanol to acetic acid using hydrogen peroxide, and compared the relevant parameters with the respective miniaturised flow process (Fig. 2, bottom). ${ }^{9}$ Furthermore, control of the flow rate and therefore of the residence time is crucial for handling highly reactive intermediates (see issue 4). In such cases, online monitoring can be achieved with various analytical tools such as IR spectroscopy.

\section{Issue 3: flow, heat and pressure-a crucial relationship}

Historically, miniaturised flow devices have been utilised for performing highly exothermic reactions that are carried out at ambient or low temperature (see issue 4). In that context safety issues are matched due to the excellent mixing and heat transfer properties of these types of reactors. ${ }^{3}$ Lately, developments in this field have focused on reactions that require more than 20 min reaction time under classical batch conditions and thus need to be heated in order to be rapidly carried out under continuous flow conditions. As a cold stream of reactants enters the reactor and residence times in a flow protocol ideally need to be in the order of a few minutes to allow high flow

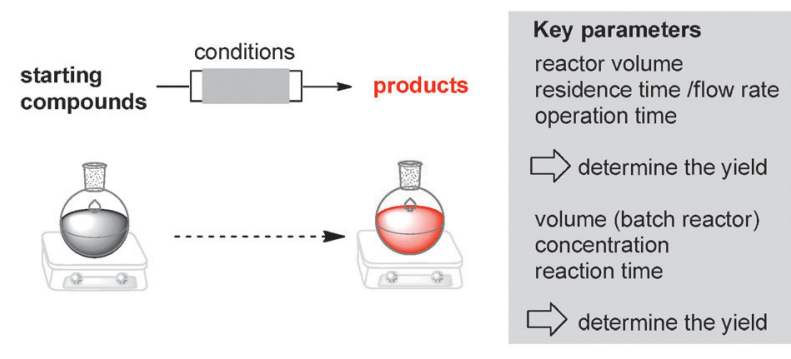

\begin{tabular}{l|cc}
\hline & $\begin{array}{c}\text { Continuous stirred } \\
\text { tank reactor } \\
\text { industrial process }\end{array}$ & $\begin{array}{c}\text { Plug flow } \\
\text { microreactor }\end{array}$ \\
\hline Residence time / s & 1760 & 3 \\
Conversion / $\%$ & $30-90$ oscillating & $>99$ \\
Reaction volume / $\mathrm{cm}^{3}$ & 2900 & 3 \\
Space-time yield / $\mathrm{h}$ & $0.7-2.0$ oscillating & 500
\end{tabular}

Fig. 2 Key parameters in flow versus batch reaction and comparative example of a batch and flow process. rates heating through the walls of a preheated reactor has to be very rapid. If "flash" heating can be guaranteed and pressure resistant microstructured flow reactors are chosen the reaction temperature can be set well above the boiling point of the solvent even up to supercritical conditions thus leading to very short reaction times. ${ }^{10}$

Several strategies have been pursued to achieve "flash" heating. Besides external ovens microwave irradiation (MW) has been combined with flow devices. This is a very practical approach because microwave chemistry is otherwise difficult to scale in a batch environment. Under flow conditions, however, only the reactor has to be heated and not the reservoirs containing the starting materials and the products. Recently, inductive heating (IH) of conductive materials and superparamagnetic nanoparticles used as fixed bed materials inside flow reactors has been added as a second "indirect" method to the portfolio of heating techniques. ${ }^{11,17}$

Kappe et al. utilised the special properties of alcohols such as ethanol at high temperature in the supercritical state to perform the esterification of benzoic acid 5 under flow conditions. ${ }^{12}$ The authors pointed out that high temperature and supercritical conditions are required as below $200{ }^{\circ} \mathrm{C}$ no esterification to ethyl ester $\mathbf{6}$ occurs.

Poliakoff and co-workers utilised supercritical water to carry out several industrially relevant conversions in a flow device. ${ }^{13}$ As one illustrative example the one pot hydrolysis followed by cyclisation of 6-aminocapronitrile (7) to furnish $\varepsilon$-caprolactam (8) is given in Scheme 2.

Microwave and flow has been combined repeatedly, ${ }^{14}$ e.g. the Dimroth rearrangement can be achieved in a flow device at $200{ }^{\circ} \mathrm{C}$ with microwave assistance. The 1,3-thiazine 9 was transformed into the corresponding 3-substituted hydropyrimidine $\mathbf{1 0} .^{15} \mathrm{~A}$ remarkable example was recently disclosed by Organ and co-workers. ${ }^{16}$ They performed the three-component coupling of terminal alkyne 13, morpholine (11), and benzaldehyde (12) to yield propargyl amine 14 under flow conditions (Scheme 3). Heating was achieved by microwave irradiation which served to specifically activate a thin coating of 


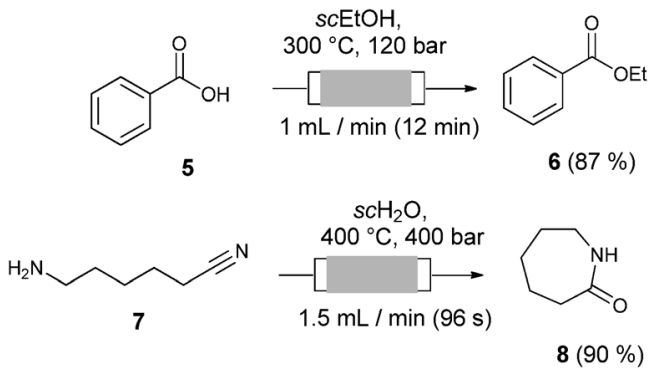

Scheme 2 Flow synthesis under supercritical conditions.

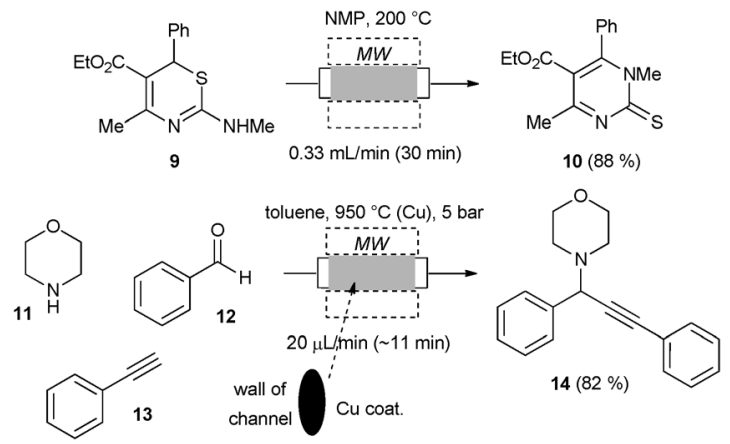

Scheme 3 High temperature-high pressure microwave-assisted synthesis in mini flow devices.

$\mathrm{Cu}$ or $\mathrm{Au}$ catalyst inside the glass capillary. By carefully monitoring the actual temperature of the glass/metal surface with the aid of a high definition

IR camera they could show that the genuine temperature was $950{ }^{\circ} \mathrm{C}$ instead of $185{ }^{\circ} \mathrm{C}$ as was originally determined for the bulk mixture. This is an

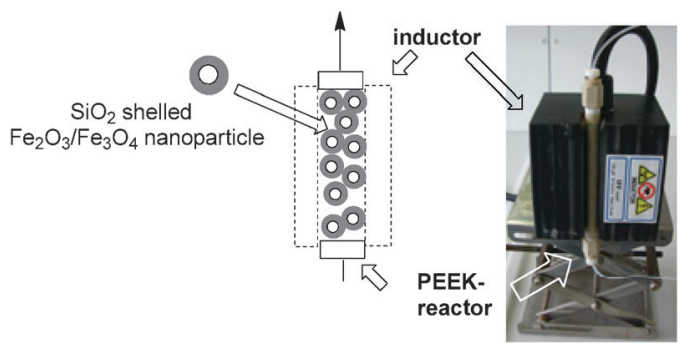

Fig. 3 Inductively heated mini flow reactors using superparamagnetic nanoparticles (MagSilica ${ }^{\mathrm{TM}}$ ).

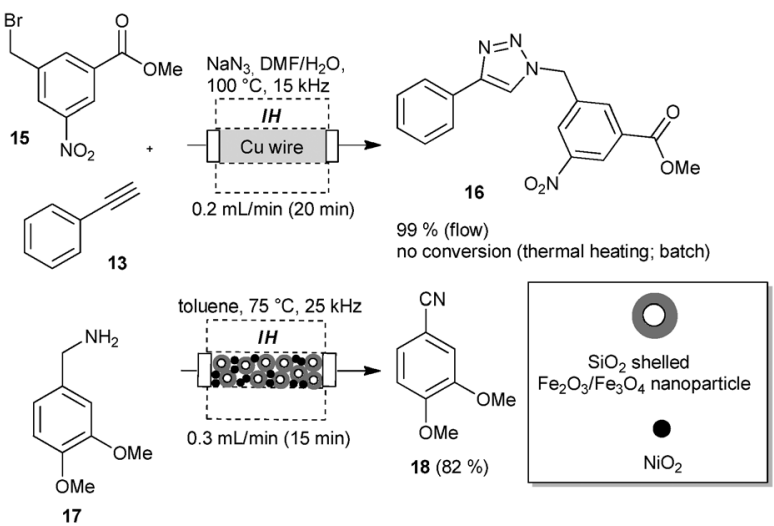

Scheme 4 Inductively heated mini flow reactions. important and remarkable finding and should apply to metal surfaces and catalytic particles in general. Kirschning et al. utilised MagSilica ${ }^{\mathrm{TM}}$ nanoparticles based on $\mathrm{Fe}_{2} \mathrm{O}_{3} / \mathrm{Fe}_{3} \mathrm{O}_{4}$ that are coated with silica and that show superparamagnetic properties (Fig. 3). ${ }^{17}$ These materials rapidly heat up by induction in the presence of an electromagnetic field of medium frequency $(25 \mathrm{kHz})$.

Used as packed bed materials inside flow devices it was demonstrated that basically all common reactions (e.g. transesterifications, condensations, Pd-catalysed cross coupling reactions and Wittig olefinations) that require heat can be performed under flow conditions. ${ }^{18}$

As an extension of this work it was demonstrated that copper wire can be heated in an inductive field and at the same time can act as a catalyst for the 1,3-dipolar cycloaddition between alkyne 13 and an azide formed in situ from benzyl bromide 15 (Scheme 4). ${ }^{19}$ Another extension of this technique employed metal oxides such as $\mathrm{CrO}_{2}$ $\left(\right.$ MagTrieve $\left.{ }^{\mathrm{TM}}\right)$ or $\mathrm{NiO}_{2}$, respectively, as additional fixed bed material in order to perform oxidations under heated flow conditions. $^{20}$ Thus, benzyl amine $\mathbf{1 7}$ was converted into aryl nitrile $\mathbf{1 8}$ by inductively heated flow synthesis. Importantly, metal leaching is very low both for iron as well as for $\mathrm{Cr}$ or $\mathrm{Ni}$ $(<4.2 \mathrm{ppm})$.

\section{Issue 4: flow and reactive intermediates-a classical application}

The excellent heat and mass transfer properties of miniaturised flow devices have made them ideal tools for carrying out synthesis with hazardous reagents or for the creation of highly reactive intermediates that commonly initiate highly exothermic reactions. Fluorinations have become classical applications in this context providing the safer production of fluorinated compounds compared to batch $_{\text {protocols. }}{ }^{21}$

Several impressive and at the same time simple setups for those reactions were published by Sandford et al. Elemental fluorine was pumped through a custom-made mini reactor (channel diameter $0.5 \mathrm{~mm}$ ) to safely fluorinate a variety of diketones such as $\mathbf{1 9}$ (Scheme 5). ${ }^{22}$ 


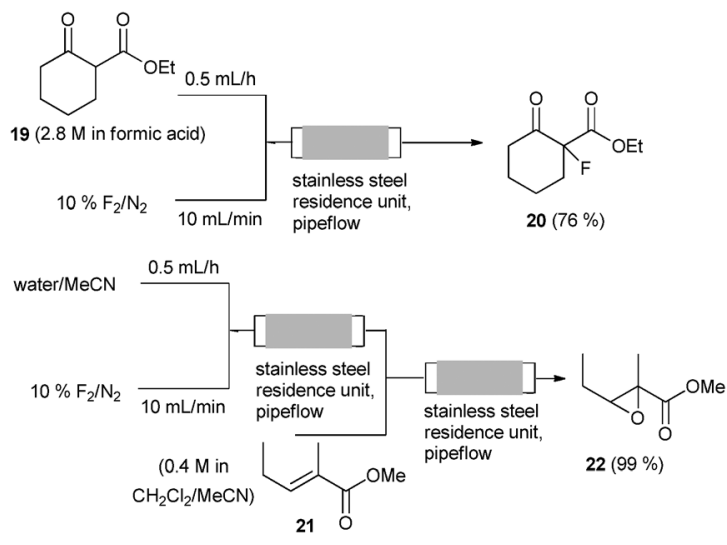

Scheme 5 Continuous flow fluorinations/epoxidations in a micro reactor.

Furthermore, they used a slightly modified device for in situ generation of highly reactive $\mathrm{HOF}^{*} \mathrm{MeCN}$ from fluorine and water and used this reagent system to efficiently epoxidise different alkenes 2 . $^{23}$

Jähnisch and co-workers developed an ozonolysis/reduction sequence and elegantly showed that it can be applied in the preparation of a vitamin D precursor $\mathbf{2 4}$ in a microplant using a five-channel mixer for the crucial liquid/ gas mixing and also for the final $\mathrm{NaBH}_{4}$ promoted reduction step. ${ }^{24}$ Importantly, an FTIR sensor was placed in between the first reactor outlet and the degassing unit which served to monitor the progress of the ozonolysis step (Scheme 6). The desired alcohol $\mathbf{2 4}$ was collected with throughputs of up to $1.2 \mathrm{mmol} \mathrm{\textrm {min } ^ { - 1 }}$ equaling $2.9 \mathrm{~g}$ per $5.5 \mathrm{~min}$ isolated material. Recently, Ley and co-workers disclosed a flow-through chemistry apparatus based on a semipermeable Teflon AF-2400 membrane which allows ozone to be introduced into a stream of alkenes to conduct ozonolysis. ${ }^{25}$

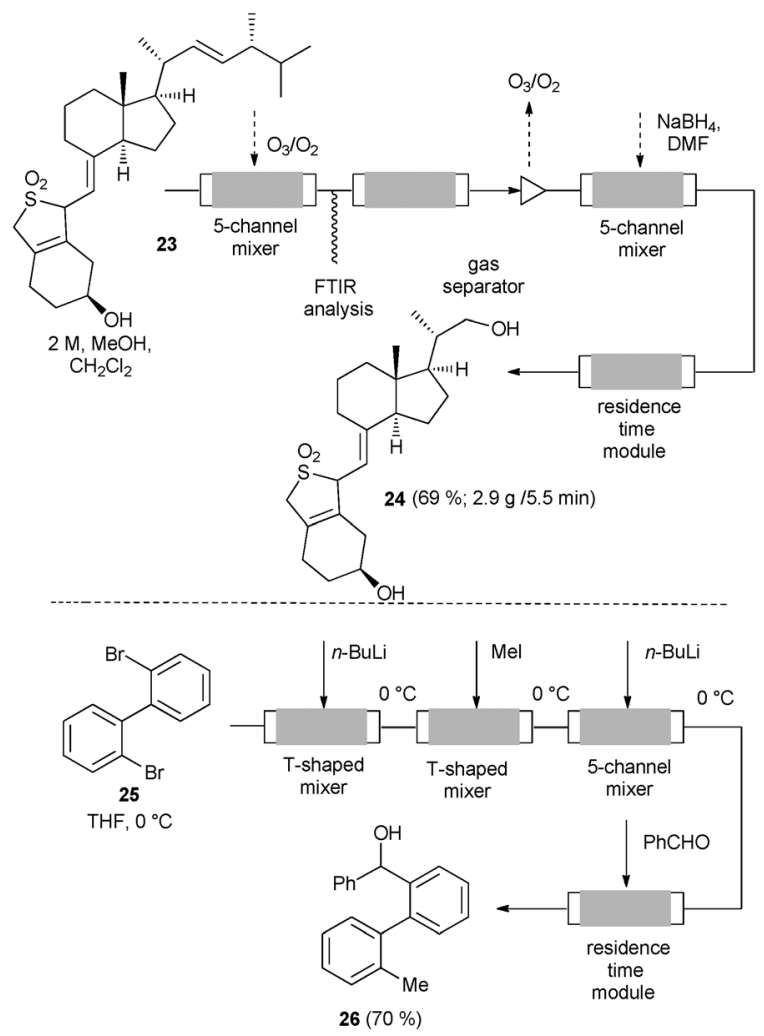

Scheme 6 Continuous flow ozonolysis-reduction sequence in micro reactors and sequential lithiations in a mini reactor.
Yoshida and co-workers reported a selective monolithiation protocol of dibromobiaryls such as $\mathbf{2 5}$ with subsequent trapping of the anion by an electrophile within a microfluidic device (Scheme 6). ${ }^{26}$ In contrast to the corresponding batch experiments it was shown that the desired monolithiated species can be generated with very high selectivity and remarkably, this protocol gave excellent results at uncommonly high temperatures such as $0{ }^{\circ} \mathrm{C}$ or rt. Based on these findings the authors developed a sequential procedure which included a second lithiation followed by the reaction with a second electrophile to yield biaryl 26. This illustrative example clearly demonstrates the benefits of heat and mass transfer in micromixing elements so that conventional low temperature batch setups with the associated lack of chemo- and regiocontrol can be avoided. It should be noted that these principal studies pave the way to safely carry out transformations involving hazardous reactants or highly reactive intermediates on a large scale, which is of relevance for industrial applications (issue 10).

\section{Issue 5: flow and supported reagents}

In addition to simple tube-like hollow reactors, packed reactors have seen wide application in industry and lately also in the synthesis of drug-like molecules. Often these fixed beds are functionalised with heterogeneous catalysts and up to date, these functionalised materials have found wide use in the automobile business. ${ }^{27}$ In general, when packing is irregular these fixed beds show uncontrolled fluid dynamics which results in various disadvantages from a chemical reaction engineering standpoint. Hot spot formation and stagnation zones need to be mentioned as well as broad residence time distribution and in essence low process efficiency.

With the development of solid phase bound homogeneous catalysts and reagents during the decade of combinatorial chemistry and high throughput synthesis the repertoire of functionalised materials available to the chemist has increased dramatically. Furthermore, additional techniques based on 
functionalised polymers or inorganic supports such as scavengers or the catch and release concept set the stage to broaden the use of flow reactors and the opportunities to perform synthesis under flow conditions. Based on the knowledge accumulated in the automobile industry it became evident that the fixed bed material ideally should be microstructured as a monolith. ${ }^{27}$ Monolithic materials have a large geometric surface area and a high void volume. As a result the pressure drop is commonly low during the passage of a gas or a fluid. ${ }^{28}$ In addition one achieves a large contact area of the reagent or the catalyst with the fluid. ${ }^{29}$

In 2001, applications of novel chemically functionalised monolithic glass/polymer composite materials inside minifluidic reactors were reported. ${ }^{30}$ Three flow steps all promoted by reagents bound to the monolithic composite material, an oxidation, desilylation and a reductive amination, afforded aminofunctionalised steroid $\mathbf{3 0}$ starting from protected testosterone 27 (Scheme 7).

Ley and co-workers reported on a Merrifield-type azide monolith incorporated into a flow cartridge which was

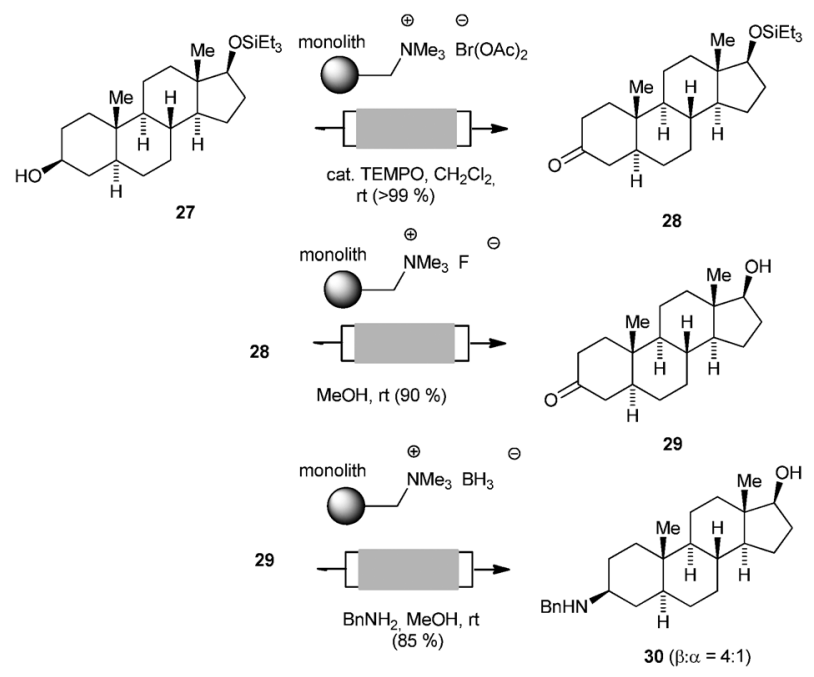

Scheme 7 Monolithic glass/polymer composite material employed in mini flow reactors.

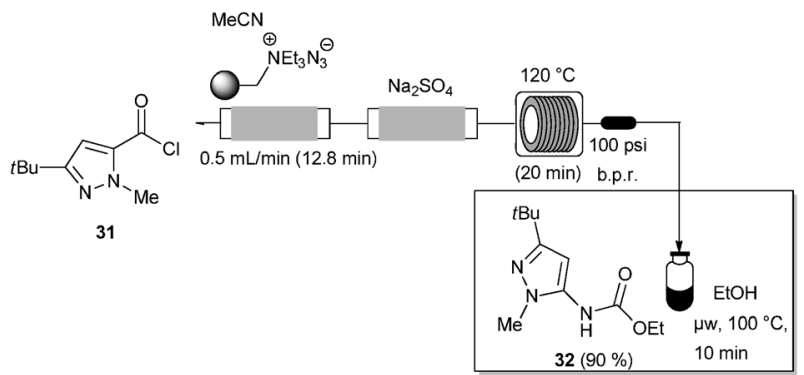

Scheme 8 Azide monoliths as mini flow reactors for Curtius rearrangement reactions.

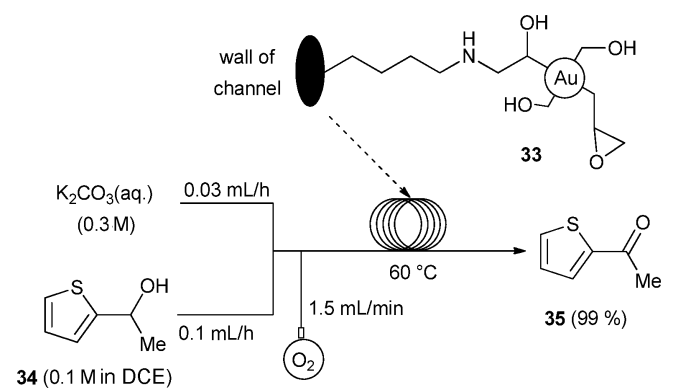

Scheme 9 Aerobic oxidation with a gold-immobilised microchannel flow reactor.

employed for the preparation of carbonyl azides from acyl chlorides 31 . $^{31}$ Under flow conditions the rearrangement to the isocyanate was initiated which was trapped with different nucleophiles such as ethanol to yield carbamates like 32 (Scheme 8).

\section{Issue 6: flow and supported catalysts-an ideal match}

In a world where sustainability concerns are a big issue especially for the chemical industry the call to perform complex reactions and avoiding wasteful isolation and purification procedures is getting louder. Thus, the immobilisation of organo- and metal catalysts in combination with flow chemistry is a possible way to address this task. ${ }^{5}$

A microchannel flow reactor with immobilised gold particles was recently described by Kobayashi and co-workers. ${ }^{32}$ In a three step procedure a polysiloxanecoated micro capillary was functionalised with microencapsulated gold particles $\mathbf{3 3}$ and was successfully utilised for the oxidation of alcohols like $\mathbf{3 4}$ to yield the corresponding carbonyl compounds like $\mathbf{3 5}$ under aerobic flow conditions (Scheme 9).

Under the reaction conditions employed, the gold-immobilised capillaries showed no loss in activity and no leaching of gold after being operated for four days.

Pericàs and co-workers have published a series of catalytic applications in miniaturised flow devices using polymer-bound chiral catalysts. ${ }^{33}$ In one example, the authors filled a jacketed omnifit column with the polystyrenesupported proline derivative $\mathbf{3 9}$ and introduced it into a flow system. ${ }^{34}$ The stereoselectivity of the Mannich-type aldol reaction of propanal (36) with imine $\mathbf{3 7}$ in a batch process could be exactly replicated in the flow process (Scheme 10).

\section{Issue 7: flow and multicomponent reactions}

Multicomponent reactions (MCRs) are particularly attractive applications for performing synthesis in microstructured flow devices. ${ }^{35}$ By mixing and heating three sometimes four different building 


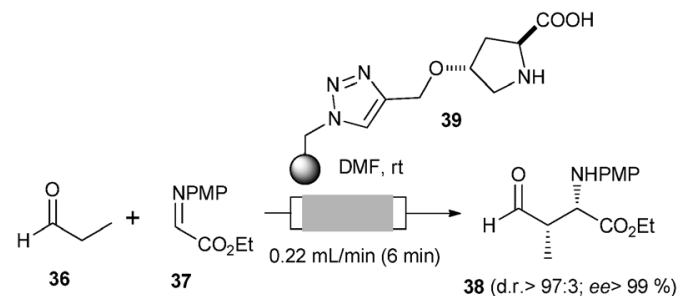

Scheme 10 Asymmetric organocatalysis in a mini flow reactor with a polymer-bound proline derivate.

blocks products are rapidly gained which are structurally already fairly complex.

Thus, by submitting a stream of $p$-chlorobenzaldehyde (40), indandione (41) and 2,6-diaminopyrimidin-4(3H)one (42) in wet DMF through a reactor filled with $\mathrm{MagSilica}^{\mathrm{TM}}$ at $100{ }^{\circ} \mathrm{C}$ the preparation of furo $\left[3^{\prime}, 4^{\prime}: 5,6\right]$ pyrido $[2,3$ d] pyrimidine $^{36} \mathbf{4 3}$ in a three-component reaction became possible (Scheme 11). Under these reaction conditions, full conversion could be achieved and the pure product $\mathbf{4 3}$ (87\% yield) crystallised upon addition of water. The corresponding batch reaction employing the same reaction conditions afforded 43 only after an extended reaction time of $3 \mathrm{~h}$ in $70 \%$ yield. $^{18}$

Furthermore, Kirschning and co-workers reported on the Petasis multicomponent

reaction. ${ }^{18}$ In their tailor-made glass reactor loaded with steel beads as heating source aldehyde $\mathbf{4 4}$, morpholine $\mathbf{1 1}$ and aryl boronic acid $\mathbf{4 5}$ were reacted to afford the Petasis product $\mathbf{4 6}$ in higher yield compared to the corresponding batch experiment (Scheme 12).

\section{Issue 8: flow and photochemistry-new options}

Despite the fact that photochemistry offers many advantages over heated reactions because of the precise control of the energy input only rarely photochemical processes have been realised on an industrial scale. One reason is that specialised reaction vessels with a light
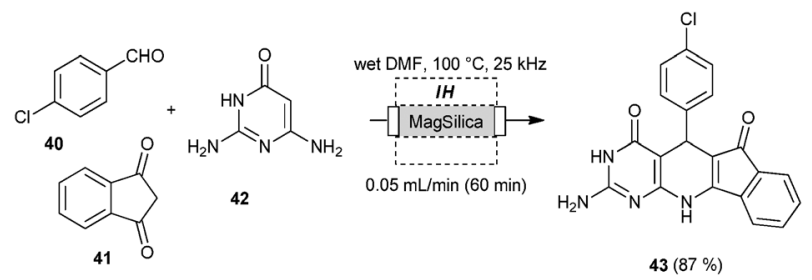

Scheme 11 An inductively heated multicomponent reaction towards complex pyrimidines under mini (meso) flow conditions.

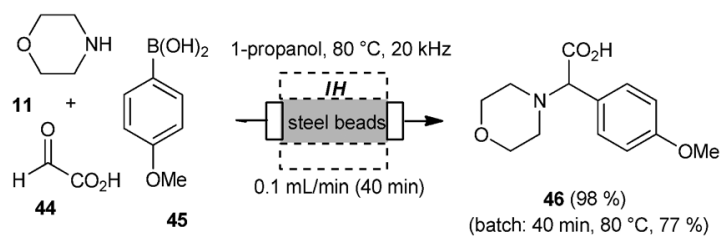

Scheme 12 Inductively heated Petasis multicomponent reaction under mini (meso) flow conditions.

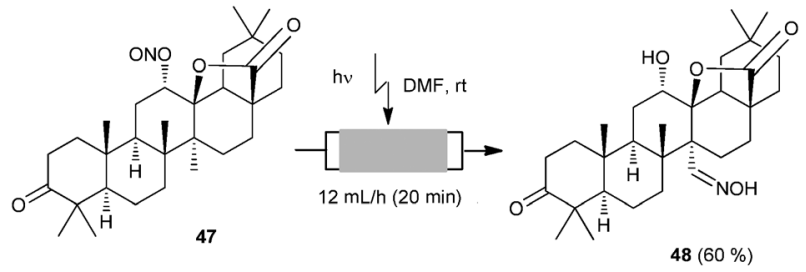

Scheme 13 Photochemical Barton reaction under mini (meso) flow conditions. source are required in which the light source is best placed in the centre of the reaction mixture. However, the design of such vessels poses a difficulty on an industrial scale. In addition these setups often generate a large amount of heat and therefore require additional cooling systems.

There is a trend to switch from batch to continuous-flow photo-processes thus circumventing some of these problems. ${ }^{37}$ Indeed, it is suggested that flow processes are far more effective for large-scale photochemical synthesis when the concept of numbering up by using several microreactors in parallel is applied. This setup ensures uniform irradiation to the entire reaction solution particularly because the depth of a microreactor is commonly small (100-1000 $\mu \mathrm{m})$, maximum penetration of light and thus irradiation even of relatively concentrated solutions can straightforwardly be achieved. Importantly, the production rate of a photochemical process can be easily increased in a microphotoreactor by raising the irradiation power of the photochemical processes. This allows to increase the flow rate of the system. As microstructured reactors possess high heat transfer coefficients cooling that may be required during a photochemical process is achieved efficiently. Further miniaturisation is possible with the use of light emitting diodes (LEDs) instead of conventional light sources. ${ }^{38}$

An illustrative example reported by the Ryu group using a Pyrex glass reactor and a $15 \mathrm{~W}$ black light is the Barton reaction of steroidal derivative 47. It yielded the bridged $\gamma$-lactone $\mathbf{4 8}$ (Scheme 13). ${ }^{39}$ In this case, it was also shown that numbering up of mesoreactors is a valid concept for large scale photochemistry. Thus, by using serially connected mesoreactors $(1000 \mu \mathrm{m}$ wide, $500 \mu \mathrm{m}$ deep and $1 \mathrm{~m}$ total length with a hold volume of $8 \mathrm{~mL}$ ) applying eight $20 \mathrm{~W}$ black lights, gram amounts of the Barton product $\mathbf{4 8}$ were prepared within $20 \mathrm{~h}$.

By combining photochemistry in microstructured reactors with the immobilisation of a catalytically active species like $\mathrm{TiO}_{2}$ doped with $\mathrm{Pt}$ (300 nm thickness) (see also issue 6) on the inner walls of the microreactor (770 $\mu \mathrm{m}$ channel width) Takei and co-workers ${ }^{40}$ recently 


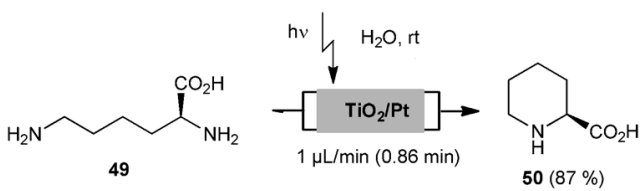

Scheme 14 Photocatalytic synthesis of L-pipecolinic acid $\mathbf{5 0}$ in a micro flow reactor.

demonstrated that L-lysine 49 undergoes a photocatalytic degradative cyclisation yielding L-pipecolinic acid $\mathbf{5 0}$ (Scheme 14). The reaction rate was about 70 times higher compared to the corresponding batch process and importantly the enantiomeric excess (ee) did not decrease.

Importantly, there have been other approaches using mini (meso) flow reactors $(2.7 \mathrm{~mm}$ inner tube diameter) making use of high concentrations $(0.1-0.4 \mathrm{M})$ and high flow rates $\left(2-10 \mathrm{~mL} \mathrm{~min}^{-1}\right)$ yielding up to $685 \mathrm{~g}$ of the product within $24 \mathrm{~h}$ operation time. ${ }^{41}$

\section{Issue 9: flow and multistep synthesis-mimicking nature}

Synthesis under flow conditions is ideally suited to perform multistep synthesis using linearly assembled flow devices. While synthesis under batch conditions means that each intermediate product of a multistep synthesis has to be isolated in bulk the situation is totally different under flow conditions. In fact, flow closely resembles Nature's concept of multistep biosynthesis where a cascade of enzymes $(a-c)$ directly transforms the product of the preceding enzymatic step. In essence all intermediates B and $\mathrm{C}$ are only present in small amount (Scheme 15). The same situation holds true in a continuously operated multistep synthesis using linearly linked flow reactors, thereby minimising work-up and isolation protocols. In addition, one can create flow setups in which two short linear synthetic flow sequences converge into one flow device which allows production of complex molecules from two fairly complex building blocks. This is a scenario less commonly found in Nature. Multistep flow synthesis may also be very advantageous when highly unstable intermediates are formed as mentioned in issue 4.

A typical example of a multistep continuous-flow process relevant for a medicinal chemistry program was recently disclosed by Ulven and co-workers

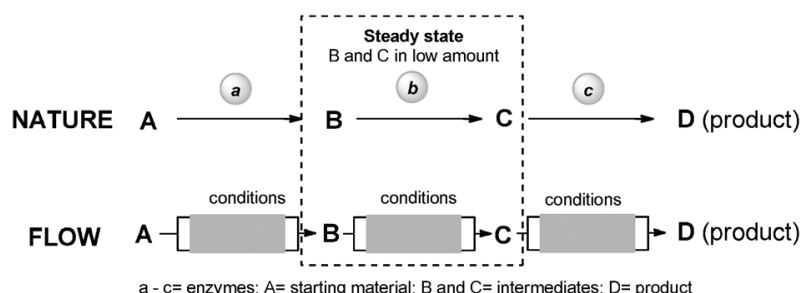

Scheme 15 Biosynthesis and multistep flow synthesis, two faces of one coin.

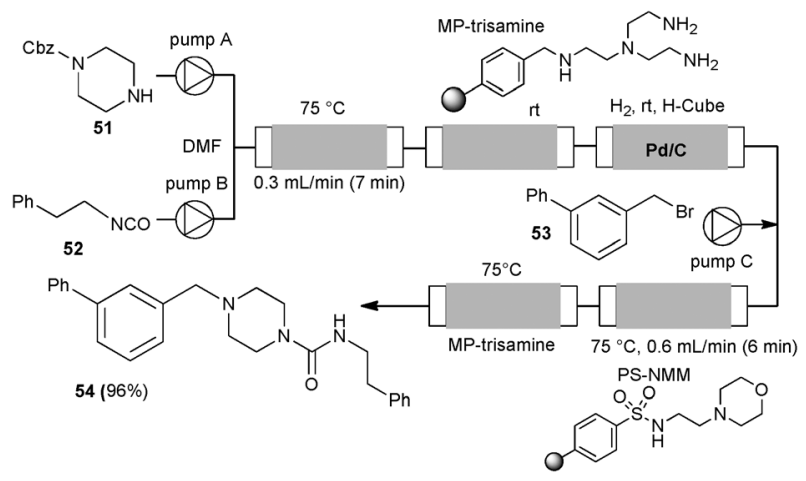

Scheme 16 Multistep flow synthesis of a piperazine library in sequentially connected mini (meso) flow reactors.

(Scheme 16). ${ }^{42}$ The preparation of a small library of piperazines $\mathbf{5 4}$ targeting chemokine receptor CCR8, which is of importance for the treatment of various inflammatory and allergic conditions, was achieved by a continuous process. During the process three variable building blocks 51-53 were assembled. A Cbz-protected diamine $\mathbf{5 1}$ was coupled with an isocyanate 52. A scavenging protocol followed by reductive removal of the $\mathrm{Cbz}$ group liberated the second amine which was alkylated with various benzyl bromides 53 followed by a second set of scavenging cartridges yielding the target piperazines 54 .

The most intriguing example of a multistep flow synthesis which closely mimicks Nature's way of multistep synthesis of complex natural products was described by Ley and co-workers (Scheme 17). ${ }^{43}$ The multistep synthesis targeted the alkaloid natural product $( \pm)$-oxomaritidine $\mathbf{6 3}$ and relied on various packed columns containing immobilised reagents, catalysts, scavengers or catch and release agents. The synthesis started with formation of azide $\mathbf{5 6}$ from benzyl bromide $\mathbf{5 5}$ which was followed by the formation of an azaWittig intermediate trapped on the supported material. In parallel, aldehyde 58 was prepared from 3,4-dimethoxybenzyl alcohol 57 and passed through the column containing the immobilised aza-Wittig intermediate, yielding the desired imine 59. Catalytic hydrogenation gave amine $\mathbf{6 0}$ which was then collected online and a solvent switch from THF to $\mathrm{CH}_{2} \mathrm{Cl}_{2}$ was performed. This step represented the only manual handling operation during the whole process. In the following, trifluoroacylation of the secondary amine $\mathbf{6 0}$ was conducted in a T-type microfluidic device at higher pressure (back pressure regulator) and the product was continuously purified by a scavenging protocol.

The reaction stream was then directed into a column containing a polymersupported (ditrifluoroacetoxyiodo) benzene (PS-PIFA) which performed the oxidative phenolic coupling to $\mathbf{6 2}$. Impressively, $( \pm)$-oxomaritidine $\mathbf{6 3}$ was collected in $90 \%$ purity after removal of the trifluoroacyl group. The authors note that all steps proceeded almost quantitatively; only the phenolic oxidation gave a moderate yield of $50 \%$, a clear proof for how efficient multistep flow processes can be conducted. 


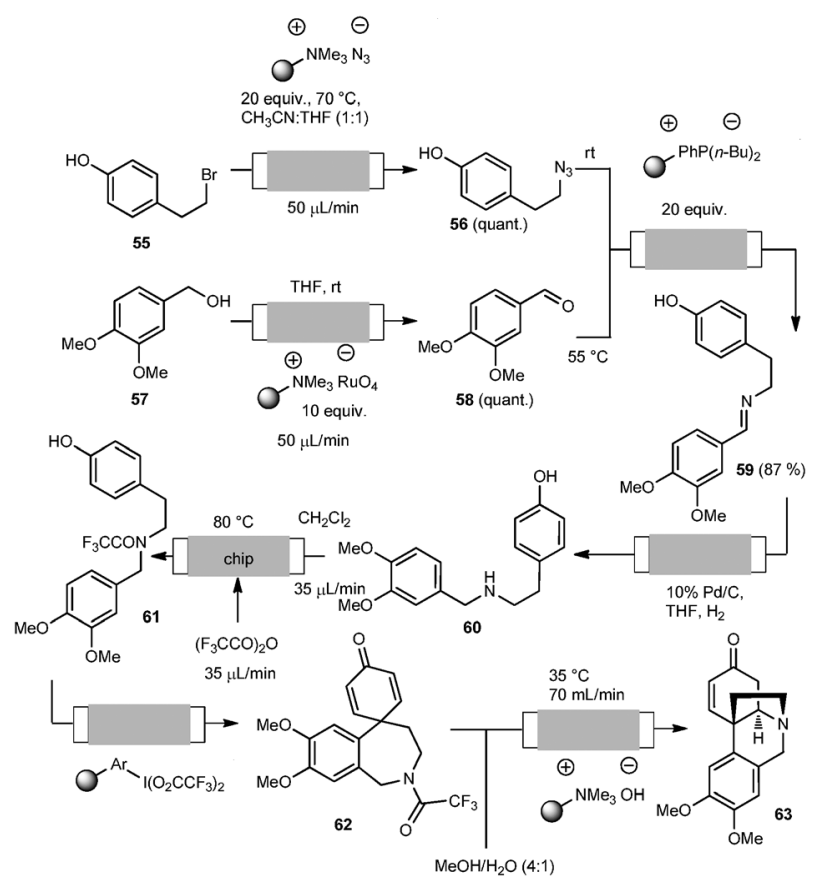

Scheme 17 Multistep flow synthesis of the alkaloid ( \pm )-oxomaritidine 63 employing mini (meso) and micro flow reactors.

\section{Issue 10: flow, scale up and industrial application- think big}

For a long time the chemical industry has relied on the continuous production of chemicals-commonly for commodities and less much so for fine chemicals and pharmaceuticals. As no flow equipment on the laboratory scale was available until recently, the chemistry developed in the laboratory was based on batch processes. This means that research was often disconnected from process chemistry which led to many problems and extra optimisations or even total redesign of the initial synthetic strategy.

With bench top flow systems now being available some of these frustrations between process and research chemists will be reduced because an established flow process is readily scalable. In fact, the volume of a flow reactor is very small compared to the scale of the whole process. Therefore, reaction parameters such as temperature, concentration, composition of reactants established for a small scale flow process can directly be transferred to larger flow reactors, without the need for substantial alteration of the conditions. ${ }^{44}$ Even if the process parameters need to be changed communication between process and research chemists is highly simplified because both now understand the philosophy of chemistry under flow conditions and are familiar with online monitoring and optimisation (see Scheme 1).

When mini (meso) flow equipment is utilised for scale-up concepts the extension of product collection time or increase of the length or size of the microchannel or tube are valid strategies (scale out).

Alternatively, the parallel operation of many identical channels ("numbering up principle") can be envisaged but from a process engineering point of view this strategy is regarded to be far from ideal because the individual flow systems must show identical flow properties and the system requires highly complex online monitoring.

A second line of development for flow chemistry is the pharmaceutical industry. In fact, mini (meso) flow systems have reached the research laboratories of medicinal chemists after two decades of combinatorial chemistry and high throughput synthesis with limited success in finding new pharmaceutical entities. The pharmaceutical industry still searches for new (enabling) technologies ${ }^{5}$ to speed up the search and optimisation of new lead structures. Current applications are multistep processes and the preparation of 50 to $100 \mathrm{mg}$ amount of new compounds as well as the automated sequential preparation of small building blocks or text compounds using one flow system.

It needs to be noted that it is far from certain whether flow technology will provide a suitable technological synthesis platform to solve the current problems in the pharmaceutical sector.

\section{Conclusions}

Flow chemistry, an old process concept from industry, has reached chemical laboratories and is now being conducted in miniaturised apparatuses in the laboratory. Undoubtedly, it will have a major impact on how we "think" and do synthesis in the future particularly when scale up by continuous operation, high temperature/high pressure synthesis as well as multistep synthesis are envisaged. Undoubtedly, this enabling technology ${ }^{5}$ has the potential to change organic chemistry in a way chromatography did about 60 years ago.

\section{Notes and references}

1 Reviews on microwave-assisted organic chemistry: (a) C. O. Kappe and D. Dallinger, Mol. Diversity, 2009, 13, 71; (b) S. Caddick and R. Fitzmaurice, Tetrahedron, 2009, 65, 3325; (c) In Microwave-Assisted Organic Synthesis, ed. P. Lidström and J. P. Tierney, Blackwell Publishing, Oxford, UK, 2005; (d) In Microwaves in Organic Synthesis, ed. A. Loupy, Wiley-VCH, Weinheim, Germany, 2nd edn, 2006.

2 (a) M. P. Dudukovic, F. Larachi and P. L. Mills, Chem. Eng. Sci., 1999, 54, 1975; (b) G. M. Whiteside, Nature, 2006, 442, 368.

3 Reviews on microreactors: (a) V. Hessel, Chem. Eng. Technol., 2009, 32, 1655; (b) K. Geyer, T. Gustafson and P. H. Seeberger, Synlett, 2009, 2382; (c) R. L. Hartman and K. F. Jensen, Lab Chip, 2009, 9, 2495; (d) X. Y. Mak, P. Laurino and P. H. Seeberger, Beilstein J. Org. Chem., 2009, 5(19); (e) C. Wiles and P. Watts, Eur. J. Org. Chem., 2008, 1655; $(f)$ T. Fukuyama, M. T. Rahman, M. Sato and I. Ryu, Synlett, 2008, 151; $(g)$ B. Ahmed-Omer, J. C. Brandt and T. Wirth, Org. Biomol. Chem., 2007, 5, 733; (h) P. Watts and C. Wiles, Chem. Commun., 2007, 433; (i) B. P. Mason, K. E. Price, J. L. Steinbacher, A. R. Bogdan and D. T. McQuade, Chem. Rev., 2007, 107, 2300; (j) J. Kobayashi, Y. Mori and S. Kobayashi, Chem.-Asian $J ., 2006,1,22 ;(k)$ W. Verboom, Chem. Eng. Technol., 2009, 32, 1695; (l) J. P. McMullen and K. F. Jensen, Annu. Rev. Anal. Chem., 2010, 3, 19; (m) K. Tanaka 
and K. Fukase, Org. Process Res. Dev., 2009, 13, 983; (n) D. M. Roberge, B. Zimmermann, F. Rainone, M. Gottsponer, M. Eyholzer and N. Kockmann, Org. Process Res. Dev., 2008, 12, 905; (o) G. Jas and A. Kirschning, Chem.-Eur. J., 2003, 9, 5708; ( $p)$ A. Kirschning and G. Jas, Immobilized Catalysts, in Top. Curr. Chem., ed. A. Kirschning, 2004, p. 209; (q) I. R. Baxendale and S. V. Ley, in New Avenues to Efficient Chemical Synthesis Emerging Technologies, ed. P. $\mathrm{H}$. Seeberger and T. Blume, Springer, Berlin, Heidelberg, 2007, p. 151; (r) S. Ceylan and A. Kirschning, in Recoverable and Recyclable Catalysts, ed. M. Benaglia, John Wiley \& Sons Ltd., 2009, ch. 13, p. 379; (s) J. Yoshida, A. Nagaki and T. Yamada, Chem.-Eur. J., 2008, 14, 7450; $(t)$ J. P. McMullen and K. F. Jensen, Annu. Rev. Anal. Chem., 2010, 3, 19.

4 See for example: K. Geyer, J. D. C. Codee and P. H. Seeberger, Chem.-Eur. J., 2006, 12, 8434.

5 A. Kirschning, W. Solodenko and K. Mennecke, Chem.-Eur. J., 2006, 12, 5972.

6 Handbook of Micro Reactors, ed. V. Hessel, J. C. Schouten, A. Renken, Y. Wang and J.-i. Yoshida, Wiley-VCH, Weinheim, 2009.

7 (a) in Microreactors in Organic Synthesis and Catalysis, ed. T. Wirth, Wiley-VCH, Weinheim, Germany, 2008, ch. 5, pp. 211-275; (b) N. Kockmann, M. Gottsponer, B. Zimmermann and D. M. Roberge, Chem.-Eur. J., 2008, 14, 7470 .

8 J. P. McMullen, M. T. Stone, S. L. Buchwald and K. F. Jensen, Angew. Chem., 2010, 39, 7230 (Angew. Chem., Int. Ed., 2010, 49, 7076).

9 V. Hessel and H. Löwe, Chem. Eng. Technol., 2005, 28, 267.

10 Review: T. Razzaq and C. O. Kappe, Chem.-Asian J., 2010, 5, 1274.

$11 \mathrm{Kunz}$ recently disclosed directly electrically heated tubular mini reactors: U. Kunz and T. Turek, Beilstein J. Org. Chem., 2009, 5(70).

12 T. N. Glasnov and C. O. Kappe, Eur. J. Org. Chem., 2009, 1321. For analogous work see: G. Anitescu, A. Deshpande and L. L. Tavlarides, Energy Fuels, 2008, 22, 1391.

13 C. Yan, J. Fraga-Dubeuil, E. GarciaVerdugo, P. A. Hamley, M. Poliakoff, I. Pearson and A. Suart Coote, Green Chem., 2008, 10, 98.

14 A. Michrowska, K. Mennecke, U. Kunz, A. Kirschning and K. Grela, J. Am. Chem. Soc., 2006, 128, 13261.

15 T. N. Glasnov, D. J. Vugts, M. M. Koningstein, B. Desai, W. M. F. Fabian,
R. V. A. Orru and C. O. Kappe, $Q S A R$ Comb. Sci., 2006, 25, 509.

16 G. Shore, W.-J. Yoo, C.-J. Li and M. G. Organ, Chem.-Eur. J., 2010, 16, 126.

17 S. Ceylan, C. Friese, C. Lammel, K. Mazac and A. Kirschning, Angew. Chem., 2008, 120, 9083 (Angew. Chem., Int. Ed., 2008, 47, 8950).

18 S. Ceylan, L. Coutable, J. Wegner and A. Kirschning, Chem.-Eur. J., 2011, 17, 1884.

19 S. Ceylan, T. Klande, C. Vogt, C. Friese and A. Kirschning, Synlett, 2010, 2009.

20 J. Wegner, S. Ceylan, C. Friese and A. Kirschning, Eur. J. Org. Chem., 2010, 4372.

21 (a) M. Baumann, I. R. Baxendale and S. V. Ley, Synlett, 2008, 2111; (b) M. Baumann, I. R. Baxendale, L. J. Martin and S. V. Ley, Tetrahedron, 2009, 65, 6611.

22 (a) R. D. Chambers, D. Holling, R. C. H. Spink and G. Sandford, Lab Chip, 2001, 1, 132; (b) R. D. Chambers, M. A. Fox and G. Sandford, Lab Chip, $2005,5,1132$.

23 C. B. McPake, C. B. Murray and G. Sandford, Tetrahedron Lett., 2009, 50, 1674.

24 S. Hübner, U. Bentrup, U. Budde, K. Lovis, T. Dietrich, A. Freitag, L. Küpper and K. Jähnisch, Org. Process Res. Dev., 2009, 13, 952.

25 M. O'Brien, I. R. Baxendale and S. V. Ley, Org. Lett., 2010, 12, 1596.

26 A. Nagaki, N. Takabayashi, Y. Tomida and Y.-i. Yoshida, Beilstein J. Org. Chem., 2009, 5(16).

27 C. D. Keith, U.S. Patent 3,441,381, Apparatus for purifying exhaust gases of an internal combustion engine, April 29, 1969.

28 (a) F. Svec and J. M. J. Frechet, Science, 1996, 273, 205; (b) D. A. Hickman and L. D. Schmidt, Science, 1993, 259, 343.

29 (a) A. Kirschning, U. Jas and G. Kunz, Chemistry in flow-new continuous flow reactors in organic synthesis, Innovation and Perspectives, in Solid Phase Synthesis and Combinatorial Libraries, ed. R. Epton, MPG Book Ltd., Kingswindford, UK, 2004; (b) F. Svec and C. G. Huber, Anal. Chem., 2006, 78, 2100.

30 A. Kirschning, C. Altwicker, G. Dräger, J. Harders, N. Hoffmann, U. Hoffmann, H. Schönfeld, W. Solodenko and U. Kunz, Angew. Chem., Int. Ed., 2001, 40, 3995 (Angew. Chem., 2001, 113, 4118).

31 M. Baumann, I. R. Baxendale, S. V. Ley, N. Nikbin and C. D. Smith, Org. Biomol. Chem., 2008, 6, 1587.

32 N. Wang, T. Matsumoto, M. Ueno, H. Miyamura and S. Kobayashi, Angew. Chem., Int. Ed., 2009, 48, 4744.
33 (a) D. Popa, R. Marcos, S. Sayalero, A. Vidal-Ferran and M. A. Pericàs, $A d v$. Synth. Catal., 2009, 351, 1539; (b) M. A. Pericàs, C. I. Herrerías and L. Solà, Adv. Synth. Catal., 2008, 350, 927; (c) E. Alza, C. Rodríguez-Escrich, S. Sayalero, A. Bastero and M. A. Pericàs, Chem.-Eur. J., 2009, 15 , 10167; (d) J. Rolland, X. C. Cambeiro, C. Rodríguez-Escrich and M. A. Pericàs, Beilstein J. Org. Chem., 2009, 5(56); (e) E. Alza, S. Sayalero, X. C. Cambeiro, R. Martín-Rapún, P. O. Miranda and M. A. Pericàs, Synlett, 2011, 464.

34 E. Alza, C. Rodríguez-Escrich, S. Sayalero, A. Bastero and M. A. Pericàs, Chem.-Eur. J., 2009, 15, 10167

35 (a) J. E. Biggs-Houk, A. Younai and J. T. Shaw, Curr. Opin. Chem. Biol., 2010, 3, 371; (b) B. B Toure and D. G. Hall, Chem. Rev., 2009, 109, 4439; (c) L. Kürti and B. Czako, Strategic Applications of Named Reactions in Organic Synthesis, Elsevier Academic Press, Burlington, MA, 2005; for flow applications, see; $(d)$ B. Khanetsky, D. Dallinger and C. O. Kappe, J. Comb. Chem., 2004, 6, 884; (e) W. S. Bremner and M. G. Organ, J. Comb. Chem., 2007, 9, 14.

36 S.-J. Tu, Y. Zhang, H. Jiang, B. Jiang, J.-Yong Zhang, R.-H. Jia and F. Shi, Eur. J. Org. Chem., 2007, 1522.

37 Reviews on photochemistry: (a) E. E. Coyle and M. Oelgemöller, Photochem. Photobiol. Sci., 2008, 7, 1313; (b) Y. Matushita, T. Ichimura, N. Ohba, S. Kumada, K. Sakeda, T. Suzuki, H. Tanibata and T. Murata, Pure Appl. Chem., 2007, 79, 1959.

38 Selected examples: (a) A. A. Lapkin, V. M. Boddu, G. N. Aliev, B. Goller, S. Polisski and D. Kovalev, Chem. Eng. $J ., 2008,136,331 ;(b)$ H. W. Chen, Y. Ku and A. Irawan, Chemosphere, 2007, 69. 184.

39 A. Sugimoto, T. Fukuyama, Y. Sumino, M. Takagi and I. Ryu, Tetrahedron, 2009, 65, 1593.

40 G. Takei, T. Kitamori and H. B. Kim, Catal. Commun., 2005, 6, 357.

41 B. D. A. Hook, W. Dohle, P. R. Hirst, M. Pickworth, M. B. Berry and K. I. Booker-Milburn, J. Org. Chem., 2005, 70, 7558-7564.

42 T. P. Petersen, A. Ritzen and T. Ulven, Org. Lett., 2009, 11, 5134.

43 I. R. Baxendale, J. Deeley, C. M. GriffithsJones, S. V. Ley, S. Saaby and G. K. Tranmer, Chem. Commun., 2006, 2566.

$44 \mathrm{~V}$. Hessel, presented at the 1st RSC/SCI Symposium on Continuous Processing and Flow Chemistry, 3rd-4th November 2010. 